\title{
THE STRUCTURE OF GROUPS WHICH ARE ALMOST THE DIRECT SUM OF COUNTABLE ABELIAN GROUPS
}

\author{
ALAN H. MEKLER
}

\begin{abstract}
The notion of being in standard form is defined for the groups described in the title of the paper which are of cardinality $\omega_{1}$. Being in "standard form" is a structural description of the group. The consequences of being in standard form are explored, sometimes with the use of additional set-theoretic axioms. It is shown that it is consistent that a large class of these groups, including every weakly $\omega_{1}$-separable $\omega_{1}-\Sigma$-cyclic group of cardinality $\omega_{1}$, can be put in standard form.
\end{abstract}

0. Introduction. In Problem 5 of Abelian groups [F], L. Fuchs asked: "In which groups can every infinite (pure) subgroup be imbedded in a direct summand of the same power?" Of course here and in our paper "group" means "Abelian group". For the purpose of this paper we will call a group a Fuchs 5 group if every countable subset is contained in a countable direct summand. So a Fuchs 5 group of cardinality $\omega_{1}$ is an example of the sort of group asked about. It is more usual to let a Fuchs 5 group be any group satisfying the hypothesis of the problem. Since we will be concerned almost exclusively with groups of cardinality $\omega_{1}$, there should be no confusion.

The first nontrivial example of a Fuchs 5 group was constructed by Hill $[\mathbf{H}]$. Hill's example was an $\omega_{1}$-separable group of cardinality $\omega_{1}$ which was $\omega_{1}-\Sigma$-cyclic. A group is $\omega_{1}$-separable if every countable subset is contained in a completely decomposable direct summand. We say a group is $\Sigma$-cyclic if it is a direct sum of cyclic groups and $\omega_{1}-\Sigma$-cyclic if every countable subgroup is $\Sigma$-cyclic. Most of the work on Fuchs 5 groups has centered on constructions of $\omega_{1}$-separable groups of cardinality $\omega_{1}$ with specified properties.

Certain of the constructions can be carried out without appealing to any additional set theoretic axioms. One of the most straightforward constructions of an $\omega_{1}$-separable $\omega_{1}$-free group is the following. (A group is $\omega_{1}$-free if every countable subgroup is free.)

Choose a stationary subset of $\lim \left(\omega_{1}\right)$ (the limit ordinals $<\omega_{1}$ ). (We will define stationary later.) For each $\delta \in S$ choose $\eta_{\delta}: \omega \rightarrow \delta$ an increasing sequence of ordinal not in $S$ whose supremum is $\delta$. Such an $\eta_{\delta}$ is called a ladder for $\delta$ and $\left(\eta_{\delta}: \delta \in S\right)$ is called a ladder system. We use the "( )" to denote a sequence and " \langle\rangle " to denote the subgroup generated. Our group $G$ will be the subgroup of

$$
\bigoplus_{\alpha<\omega_{1}} \mathbf{Q} x_{\alpha+1} \oplus \bigoplus_{\alpha \in S} \mathbf{Q} x_{\delta}
$$

Received by the editors July 7, 1986.

1980 Mathematics Subject Classification (1985 Revision). Primary 20K20, 20K25, 03E75; Secondary 03E35.

Research supported by NSERC Grant A8948. 
generated by

$$
\left\{x_{\alpha+1}: \alpha<\omega_{1}\right\} \cup\left\{z_{\delta, n}: \delta \in S, n<\omega\right\}
$$

where

$$
z_{\delta, n} \stackrel{\text { def }}{=}\left(x_{\delta}-\sum_{i=0}^{\eta} 2^{i} x_{n_{\delta}(i)}\right) / 2^{n+1} .
$$

It is easy ( $\mathrm{cf}$. [E2]) to see that $G$ is $\omega_{1}$-separable and $\omega_{1}$-free. One can vary the isomorphism type of $G$ by changing $S$, the quotient groups $G_{\delta+1} / G_{\delta}$ (where $G_{\alpha}=$ $\left\langle x_{\beta}: \beta<\alpha\right\rangle_{*}$ ) or the $\eta_{\delta}$ 's (cf. $[\mathbf{E 2}, 11.1]$ ). Of course, $\langle X\rangle_{*}$ denotes the pure subgroup generated by $X$. We will call groups like the above "classical" and show it is consistent that all $\omega_{1}$-separable $\omega_{1}$-free groups of cardinality $\omega_{1}$ are classical.

Other constructions of $\omega_{1}$-separable groups have concentrated on pathological properties. These constructions have all used $\diamond$-like principles which although consistent with ZFC are not provable.

The notions of being "a Fuchs 5 group" or being " $\omega_{1}$-separable" have a "weak" counterpart. We say a group is a weak Fuchs 5 group iff every countable subset $X$ is contained in a countable subgroup $B$ which is a direct summand of every countable extension. (Such a subgroup $B$ is called $\omega_{1}$-pure.) We can similarly define weakly $\omega_{1}$-separable. These "weak" notions have an interest from a logical point of view.

0.1 THEOREM [E1]. A group is $L_{\infty \omega_{1}}$-equivalent to a free group iff it is weakly $\omega_{1}$-separable and $\omega_{1}$-free. If a group is $L_{\infty \omega_{1}}$-equivalent to a direct sum of countable groups then it is a weak Fuchs 5-group.

Actually Eklof only proved the first statement. The second follows immediately from the methods in [E1]. From Shelah's work on the Whitehead problem it is known that it is independent of ZFC whether or not weakly $\omega_{1}$-separable $\omega_{1}$-free groups of cardinality $\omega_{1}$ are $\omega_{1}$-separable. (See the comments after Corollary 3.3.) It is possible to show using $\diamond$ that there are weakly $\omega_{1}$-separable groups which are indecomposable [EM].

In fact the pathological examples available assuming $\diamond$ seem to show that there is no hope of answering Problem 5 even for the best behaved class of groups, the $\omega_{1}$-separable $\omega_{1}$-free groups of cardinality $\omega_{1}$. At least this is the case if we restrict ourselves to theorems which can be proved in ZFC. The first indication that something positive could be proved concerning Problem 5 came in a remarkabie paper [E3] by Eklof called "The structure of $\omega_{1}$-separable groups". There he characterizes, under the assumption of $\mathrm{MA}+\neg \mathrm{CH}$, isomorphism of $\omega_{1}$-separable $\omega_{1}$-free groups of cardinality $\omega_{1}$. This characterization is used to obtain structural results about the direct sum decompositions of these groups. In [EM2] we were able to extend the characterization, again using $\mathrm{MA}+\neg \mathrm{CH}$, to $\omega_{1}$-separable $p$-groups. But we were not able to prove the same strong decomposition results.

This paper was inspired by [E3]. We wanted to extend his results to a wider class of Fuchs 5 groups and to give a structural description of these groups. In particular we wanted to show that only $\omega_{1}$-separable $\omega_{1}-\Sigma$-cyclic groups of cardinality $\omega_{1}$ which could be constructed without additional set theoretic assumptions were the "classical" ones. More exactly we wanted to show it is consistent with ZFC that any $\omega_{1}$-separable $\omega_{1}-\Sigma$-cyclic group of cardinality $\omega_{1}$ can be put in a certain simple form. For these purposes $\mathrm{MA}+\neg \mathrm{CH}$ is not sufficient. We used a version of the 
proper forcing axiom (PFA). This axiom is strictly stronger than MA $+\neg \mathrm{CH}$. So we will have Eklof's results as consequences, albeit with a stronger hypothesis.

Although we will recapitulate some facts about proper forcing, we have made no attempt to present a clear exposition of PFA and the related concepts. In [M3] we gave an introduction to the Proper Forcing Axiom designed for the abelian group theorist. We will assume the reader is familiar with [M3] or some other exposition of proper forcing (e.g. [D] or of course [S2]). In [M3] we announced many of the results in this paper. Indeed it was written as a companion to the present paper. Only in $\S 3$, do we require knowledge of the Proper Forcing Axiom. The other sections can be read by, and we hope will be of interest to, a wider audience.

In $\S 1$, we define what it means for a group to be in standard form. To help explain this notion we will show for $\omega_{1}$-separable $\omega_{1}$-free groups that being in standard form is equivalent to being classical. However we do not view this as a justification of the notion of standard form. We think it is most natural to construct Fuchs 5 groups as groups in standard form. Unlike other methods there is no difference between the torsion and torsion free case when one constructs $\omega_{1}$-separable $\omega_{1}-\Sigma$-cyclic groups in standard form. To illustrate the methods for the reader unfamiliar with [M1], we construct in $\S 4$ a mixed $\omega_{1}$-separable $\omega_{1}$ - $\Sigma$-cyclic group. It is possible to generalize the notion of standard form to $\omega_{n}$-separable groups of cardinality $\omega_{n}$. In [M1] we constructed $\omega_{n}$-separable $\omega_{n}$-free groups of cardinality $\omega_{n}$. These groups are constructed in the analogue of standard form.

In $\S 2$, we investigate the consequences of being in standard form. We show, assuming $\mathrm{MA}+\neg \mathrm{CH}$, that any Fuchs 5 group in standard form has a strong decomposition property. Before we can state this result we recall some definitions.

A subset $C \subseteq \omega_{1}$ is a c.u.b. if it is closed (i.e. for all $Y \subseteq C$ if $\sup Y<\omega_{1}$ then $\sup Y \in C$ ) and $C$ is unbounded (i.e. $\sup C=\omega_{1}$ ). A subset $S \subseteq \omega_{1}$ is stationary if $S \cap C \neq \varnothing$ for all cubs $C$. Further we have an equivalence relation on stationary sets namely $S \equiv S^{\prime}$ if there is a cub $C$ such that $S \cap C=S^{\prime} \cap C$. As usual we let $\tilde{E}$ denote the equivalence class of $E, 1$ denote $\tilde{\omega}_{1}$ and 0 denote $\tilde{\phi}$.

Suppose now $A$ is a weak Fuchs 5 group and $|A|=\omega_{1}$. Choose $\omega_{1}$-filtration of $\left(A_{\alpha}: \alpha<\omega_{1}\right)$ of $A$ such that for all $\alpha<\omega_{1} A_{\alpha+1}$ is $\omega_{1}$-pure. (Recall $\left(A_{\alpha}: \alpha<\omega_{1}\right)$ is an $\omega_{1}$-filtration iff $A=\bigcup A_{\alpha}$; for all $\alpha,\left|A_{\alpha}\right|=\omega$; for $\alpha<\beta, A_{\alpha} \subseteq A_{\beta}$; and for limit ordinals $\lambda, A_{\lambda}=\bigcup_{\alpha<\lambda} A_{\alpha}$.) Define $\Gamma(A)=\tilde{E}$ where $E=\left\{\alpha: A_{\alpha}\right.$ is not $\omega_{1}$-pure $\}$. As usual one can show $\tilde{E}$ is an invariant of $A$ and does not depend on the $\omega_{1}$-filtration of $A$. Also standard is the fact that $A$ is a direct sum of countable groups iff $\Gamma(A)=0$.

We prove (cf. Theorem 2.4)

THEOREM. Assume MA $+\neg \mathrm{CH}$. Suppose $A$ is a Fuchs 5 group in standard form and $\Gamma(A)=\tilde{E}$. Whenever $E=E_{1} \cup E_{2}$ (disjoint), there are $A_{1}, A_{2} \subseteq A$ so that $\Gamma\left(A_{i}\right)=\tilde{E}_{i}$ and $A=A_{1} \oplus A_{2}$.

Eklof [E3] has shown it is consistent with MA $+\neg \mathrm{CH}$ that there are $\omega_{1}$-separable $\omega_{1}$-free groups of cardinality $\omega_{1}$ which do not satisfy the theorem above. So our main theorem cannot be proved using $\mathrm{MA}+\neg \mathrm{CH}$. $\S 3$ is devoted to proving our main theorem. 
THEOREM. Assume $\mathrm{PFA}^{-}$. If $A$ is a weak Fuchs 5 group of cardinality $\omega_{1}$ and either

(I) $\Gamma(A) \neq 1$, or

(II) $A$ is $\omega_{1}-\Sigma$-cyclic,

then $A$ can be put in standard form.

1. Standard form and the classical groups. Suppose $A$ is a weak Fuchs 5 group of cardinality $\omega_{1}$ and $\left(A_{\alpha}: \alpha<\omega_{1}\right)$ is an $\omega_{1}$-filtration of $A$.

Let $E=\left\{\alpha: A_{\alpha}\right.$ is not $\omega_{1}$-pure $\} . B=\left\{B_{\alpha \tau}: \alpha \leq \tau<\omega_{1}\right.$ and $\left.\alpha \notin E\right\}$ is a system of complementary summands (w.r.t. $\left.\left(A_{\alpha}: \alpha<\omega_{1}\right)\right)$ if for all $\alpha, \rho, \tau$ for which these expressions make sense: (i) $A_{\tau}=A_{\alpha} \oplus B_{\alpha \tau}$; (ii) $B_{\alpha \tau}=B_{\alpha \rho} \oplus B_{\rho \tau}$ and (iii) if $\lambda$ is a limit ordinal then $B_{\alpha \lambda}=\bigcup_{\alpha<\rho<\lambda} B_{\alpha \rho}$. Note that a system of complementary summands witnesses that $A$ is a Fuchs 5 group. If $\alpha \notin E$, then $A=A_{\alpha} \oplus \bigcup_{\alpha<\rho} B_{\alpha \rho}$.

Definition. Suppose $A$ is a Fuchs 5 group of cardinality $\omega_{1}$. Then $A$ can be put in standard form if there are an $\omega_{1}$-filtration $\left(A_{\alpha}: \alpha<\omega_{1}\right)$, a system $B=$ $\left(B_{\alpha \tau}: \alpha<\tau, \alpha \in E\right)$ and $y=\left(Y_{\delta}: \delta \in E\right)$ (where $E=\left\{\alpha: A_{\alpha}\right.$ is not $\omega_{1}$-pure $\}$ ) such that for all $\delta \in E: A_{\delta+1}=\left\langle A_{\delta} \cup Y_{\delta}\right\rangle$; and for all $y \in Y_{\delta}$ and $\alpha<\delta, \alpha \notin E$ there is a finite set $S \subseteq \delta-E$ so that $\Pi_{0 \alpha}(y)=\sum_{\sigma \in S} \Pi_{\sigma \sigma+1}(y)$. Here for $\sigma, \rho \notin E$ $\Pi_{\sigma \rho}$ is the projection of $A$ on $B_{\sigma \rho}$ along $A_{\sigma} \oplus \bigcup_{\rho<\tau} B_{\rho \tau}\left(A_{0}\right.$ is assumed to be 0$)$.

In order to explain this definition and to justify our assertion that the $\omega_{1}$ separable $\omega_{1}$-free groups in standard form are exactly the ones with a simple construction, let us define another class of groups.

DEFinition. An $\omega_{1}$-free group $A$ is classical if for some set $S \subseteq \lim \left(\omega_{1}\right)$, $\left(\eta_{\delta}: \delta \in S\right)$ a ladder system for $S$ (assume $\eta_{\delta}(0)=0$ for all $\left.\delta\right), A$ is the subgroup of

$$
\bigoplus_{\substack{\alpha<\omega_{1} \\ \alpha \notin S}} \bigoplus_{n \in \omega} \mathbf{Q} x_{\alpha n} \oplus \bigoplus_{\delta \in S} \bigoplus_{n<n(\delta) \leq \omega} \mathbf{Q} y_{\delta n}
$$

generated by $\left\{x_{\alpha+1, n}: \alpha<\omega_{1}, n<\omega\right\} \cup\left\{y_{\delta, n}: \delta \in S\right.$ and $\left.n<n(\delta)\right\} \cup\left\{z_{\delta, n, m}: \delta \in\right.$ $S, n<\omega, m<m(\delta, n)<\omega\}$ where for each $\delta, n$ and $i<n$ there is $t(\delta, n, i)$ a linear combination of the $\left\{x_{\alpha+1, m}: \eta_{\delta}(n-1) \leq \alpha+1<\eta_{\delta}(n)\right\}$ and each $z_{\delta, n, m}$ is of the form

$$
w\left(y_{\delta, 0}-\sum_{k \leq n} t(\delta, k, 0), \ldots, y_{\delta, n}-\sum_{k \leq n} t(\delta, k, n) / d\right)
$$

where $w$ is a term and for all $s>n d \mid w(t(\delta, s, 0), \ldots, t(\delta, s, n))$ in the group generated by $\left\{x_{\alpha+1, n}: \alpha<\omega_{1}, n<\omega\right\}$.

The example we gave in the introduction is a classical group. Also we could have dropped the requirement that $A$ be $\omega_{1}$-free but then we would have had to put more complicated conditions on the $z_{\delta, n, m}$ 's to guarantee that $A$ is $\omega_{1}$-free.

1.1 PROPOSITION. An $\omega_{1}$-free group of cardinality $\omega_{1}$ is classical iff it can be put in standard form.

ProOF. Assume that $A$ is classical and let $x_{\alpha+1, n}, y_{\delta, n}, z_{\delta, n, m}, t(\delta, k, i), \eta_{\delta}$ be as in the definition of a classical group. Let $A_{\alpha}=\left\langle\left\{x_{\beta, n}: \beta<\alpha, n<\omega\right\} \cup\right.$ $\left.\left\{y_{\delta, n}: \delta<\beta, \delta \in S, n<n(\delta)\right\}\right\rangle_{*}$. So $\left(A_{\alpha}: \alpha<\omega_{1}\right)$ is an $\omega_{1}$ filtration of $A$. Rather than defining $B$ we will define the projections $\Pi_{0 \alpha}: A \rightarrow A_{\alpha}$ for $\alpha \notin S$. This will define $B_{\alpha \nu}$ to be $\operatorname{ker} \Pi_{0 \alpha} \cap A_{\nu}$. If for some $\alpha \in S, A_{\alpha}$ is $\omega_{1}$-pure, then we 
can choose a projection $\Pi$ of $A_{\alpha+1}$ on $A_{\alpha}$ and let $\Pi_{0 \alpha}=\Pi \circ \Pi_{0 \alpha+1}$. Of course $\Pi_{0 \alpha} \mid A_{\alpha}=\mathrm{id}$. Then for $\beta \geq \alpha$ let $\Pi_{0 \alpha}\left(x_{\beta n}\right)=0$. This induces a unique function on the subgroup of $A$ generated by $\left\{x_{\beta+1 n}: \beta<\omega_{1}, n<\omega\right\}$. Next if $\delta \in S$ we define $\Pi_{0 \alpha}\left(y_{\delta, n}\right)=\Pi_{0 \alpha}\left(\sum_{i \leq k} t(\delta, i, n)\right)$ where $n_{\delta}(k)>\alpha$. Our definition of being classical was designed so that $\Pi_{0 \alpha}$ extends to a unique function from $A$ to $A_{\alpha}$. Finally we let

$$
Y_{\delta}=\left\{y_{\delta, n}: n<n(\delta)\right\} \cup\left\{z_{\delta, n, m}: n<\omega, m<m(\delta, n)\right\} .
$$

Now suppose $A$ is a standard form and $\left(A_{\alpha}: \alpha<\omega_{1}\right), B=\left\{B_{\alpha \nu}: \alpha \in E\right\}$ and $y=\left\{y_{\delta}: \delta \in E\right\}$ are as in the definition of standard form. There is no difficulty in assuming $Y_{\delta}$ is a pure subgroup of $A$ and for each $y \in Y_{\delta}$, and $\alpha<\delta(\alpha \notin E)$, $y-\Pi_{0 \alpha}(y) \in Y_{\delta}$. By considering a cub subset of $\omega_{1}$ we can assume each $B_{\alpha \alpha+1}$ is a countably (infinitely) generated free group. Let $\left\{x_{\alpha n}: n<\omega\right\}$ be a set of free generators for $B_{\alpha \alpha+1}$. Choose $\left(\eta_{\delta}: \delta \in E\right)$ a ladder system. Next consider $\delta \in E$, we claim we can find $\left\{y_{\delta, n}: n<n(\delta) \leq \omega\right\}$ such that $A_{\delta+1}=\left\langle A_{\delta} \cup\left\{y_{\delta, n}: n<\right.\right.$ $n(\delta)\}\rangle_{*}$, the $y_{\delta, n}$ 's are independent modulo $A_{\delta},\left\{y_{\delta, m}: m \geq n\right\}$ is pure independent modulo $A_{\eta_{\delta}(n)}+\left\langle y_{\delta, m}: m<n\right\rangle$.

We need only consider the case $n(\delta)=\omega$. For simplicity $y$ will drop the subscript $\delta$. Choose the $y_{n}$ 's inductively. First choose $\left\{y_{n}^{0}: n<\omega\right\}$ pure independent so that $A_{\delta+1}=\left\langle A_{\delta} \cup\left\{y_{n}^{0}: n<\omega\right\}\right\rangle_{*}$ and $\left\{y_{n}^{0}: n<\omega\right\}$ are independent modulo $A_{\delta}$. Suppose that $\left\{y_{n}^{i}: n<\omega\right\}$ has been defined. Let $y_{n}^{i+1}=y_{n}^{i}$, for $n \leq i$. Next choose $y_{n}^{i+1}$ 's by induction on $n \geq i+1$ so that $\left\{y_{n}^{i+1}: n \geq i+1\right\}$ freely generate a complementary summand of $\left\langle y_{m}^{i}-\Pi_{0 \eta_{\delta}(i+1)}\left(y_{m}^{i}\right): m \leq i\right\rangle_{*}$ in $\left\langle y_{n}^{i}-\Pi_{0 \eta_{\delta}(i+1)}\left(y_{n}^{i}\right): n\langle\omega\rangle_{*}\right.$, and for all $n<\omega,\left\{y_{m}^{i}: m \leq n\right\} \subset\left\langle A_{\eta_{\delta}(i+1)} \cup\left\{y_{m}^{i+1}: m \leq n\right\}\right\rangle$. Let $y_{n}=y_{n}^{n}$. It should be clear that $\left\{y_{n}: n<\omega\right\}$ has all the desired property except perhaps that $\left\{y_{n}: n \geq m\right\}$ is pure independent modulo $A_{\eta_{\delta}(m)}$. Consider $\sum_{k=m}^{n} c_{i}-y_{i}$ and suppose that $d$ divides this sum modulo $A_{\eta_{\delta}(n)}+\left\langle y_{\delta, m}: m<n\right\rangle$. Then by the choice of $y_{n}\left(=y_{n}^{n}\right) d \mid c_{n}$. So $d \mid \sum_{i=m}^{n-1} c_{i} y_{i}$ and we are done by downward induction.

Now we can define $t(\delta, i, n)$ to be $\Pi_{\eta_{\delta}(i-1)} \eta_{\delta}(i) y_{\delta n}$ and similarly define the $z_{\delta, n, m}$ 's to be a set of generator of the (free) group $\left\langle y_{\delta, m}-\Pi_{0 \eta_{\delta}(n)}\left(y_{\delta, m}\right): m \leq n\right\rangle_{*}$.

It seems that it is also possible to give such a concrete realization of $\omega_{1}$-separable primary groups. However we view the condition that a group is in standard form as equally natural as any other condition.

2. Consequences of being in standard form. There are several natural questions about Fuchs 5 groups of cardinality $\omega_{1}$ in standard form. Are there any non-d.s.c. ones? Are all groups in standard form? Can we draw any further conclusions from knowing that a group is in standard form? As we explained in the introduction most constructions of Fuchs 5 groups are in fact constructions of groups in standard form. There is at least one construction in [E3] of an $\omega_{1}$-separable group of cardinality $\omega_{1}$ which is not in standard form. He did not construct the group for this purpose but we will show his group is not in standard form. Of course this construction cannot be carried out in ZFC. Whether or not being in standard form has any consequences outside of its intrinsic interest is a delicate question. We are only aware of two results in ZFC.

2.1 THEOREM. If $A$ is an $\omega_{1}$-separable $\omega_{1}$-free group in standard form and $\Gamma(A) \neq 1$, then $A \cong \operatorname{Hom}(B, Z)$ for some group $B$. 
A proof of this result should appear in a forthcoming work on the structure of Hom by Eklof and the author.

The second application concerns $p^{\omega+1}$-projective groups. A separable $p$-group $G$ is $p^{\omega+1}$-projective iff there is some subgroup $P \subseteq G[p](=\{x: p x=0\})$ so that $G / P$ is $\Sigma$-cyclic. (This is actually characterization of $p^{\omega+1}$-projective, cf. [FI].) A countable group is $p^{\omega+1}$-projective iff it has length $\leq \omega+1$.

2.2 THEOREM. Suppose $A$ is an $\omega_{1}$-separable $p$-group in standard form, where $\left(A_{\nu}: \nu<\omega_{1}\right),\left(B_{\nu \tau}: \nu<\rho, \nu \notin E\right)$ and $\left(Y_{\delta}: \delta \in E\right)$ witness $A$ is in standard form. $A$ is $p^{\omega+1}$-projective iff for some cub $C \subseteq \omega_{1}$ and all $\nu \in C, A_{\nu+1} / A_{\nu}$ is $p^{\omega+1}$-projective.

ProOF. First we do the easy direction. Suppose $A$ is $p^{\omega+1}$-projective. Let $P \subseteq A[p]$ be such that $A / P$ is $\Sigma$-cyclic. Since $A_{\nu}+P / P$ is an $\omega_{1}$-filtration of $A / P$, there is a cub $C$ such that for all $\nu \in C A_{\nu}+P / P$ is a direct summand of $A$. So for $\nu \in C$,

$$
\begin{aligned}
\left(A_{\nu+1} / A_{\nu}\right) /( & \left.A_{\nu}+\left(A_{\nu+1} \cap P\right) / A_{\nu}\right) \\
& \cong A_{\nu+1} /\left(A_{\nu}+\left(A_{\nu+1} \cap P\right)\right) \cong A_{\nu+1}+P / A_{\nu}+P \subseteq A / A_{\nu}+P
\end{aligned}
$$

which is $\Sigma$-cyclic. Since $A_{\nu}+\left(A_{\nu+1} \cap P\right) / A_{\nu} \subseteq\left(A_{\nu+1} / A_{\nu}\right)[p], A_{\nu+1} / A_{\nu}$ is $p^{\omega+1}$ projective.

Now suppose $A_{\nu+1} / A_{\nu}$ is $p^{\omega+1}$-projective for all $\nu$ in a cub. By changing the filtration we can assume $A_{\nu+1} / A_{\nu}$ is $p^{\omega+1}$-projective for all $\nu$. For $\nu, \tau \notin E$, let $\Pi_{\nu \tau}$ be the projection of $A$ on $B_{\nu \tau}$ along $A_{\nu} \oplus \bigcup_{\rho>\tau} B_{\tau \rho}$. We define $P$ by induction. Let $P_{0}=\{0\}$. If $\nu \notin E$, let $P_{\nu+1}=P_{\nu} \oplus B_{\nu \nu+1}[p]$. For limit ordinals $\lambda$, let $P_{\lambda}=\bigcup_{\nu<\lambda} P_{\nu}$.

Suppose $\delta \in E$. $Y_{\delta}$ can be chosen to be a subgroup so that $y \in Y_{\delta}, \nu<\delta$ and $\nu \notin E$ implies $y-\Pi_{0 \nu}(y) \in Y_{\delta}$. So for $y \in Y_{\delta}$ if $p y \in A_{\delta}$ and so in $A_{\nu}$ for some $\nu<\delta, \nu \notin E$ then $p\left(y-\Pi_{0 \nu}(y)\right)=0$. Hence it can be assumed that for all $x \in\left(A_{\delta+1} / A_{\delta}\right)[p]$ there is $y \in Y_{\delta}[p]$ so that $x \in y+A_{\delta}$. Choose $Q \subseteq\left(A_{\delta+1} / A_{\delta}\right)[p]$ so that $\left(A_{\delta+1} / A_{\delta}\right) / Q$ is $\Sigma$-cyclic. Then there is $Q_{\delta} \subseteq Y_{\delta}[p]$ so that $Q_{\delta}+A_{\delta} / A_{\delta}=Q$ and $\left(Q_{\delta}+P_{\delta}\right) \cap A_{\delta}=P_{\delta}$. To see such a $Q_{\delta}$ exists, first pick an independent set of generators for $Q$ and then choose preimages. Let $P_{\delta+1}=Q_{\delta}+P_{\delta}$.

Let $P=\bigcup_{\nu<\omega_{1}} P_{\nu}$. It is clear that $P \cap A_{\nu}=P_{\nu}$. It remains to see that $A / P$ is $\Sigma$-cyclic. By induction on $\nu$ we will show:

(i) $A_{\nu} / P_{\nu}$ is $\Sigma$-cyclic,

(ii) $A_{\nu+1} /\left(A_{\nu}+P_{\nu+1}\right)$ is $\Sigma$-cyclic; and

(iii) $\left(A_{\nu}+P_{\nu+1}\right) / P_{\nu+1}$ is pure in $A_{\nu+1} / P_{\nu+1}$.

The only difficult case occurs when $\delta \in E$ and we must verify (i) for $\delta+1$ and (ii) and (iii) for $\delta$. The choice of $Q_{\delta}$ ensures that $A_{\delta+1} /\left(A_{\delta}+P_{\delta+1}\right)$ is $\Sigma$-cyclic. Next we show $\left(A_{\delta}+P_{\delta+1}\right) / P_{\delta+1}$ is pure in $A_{\delta+1} / P_{\delta+1}$. Suppose $p^{n} \mid a+x+y$ where $a \in A_{\delta}, x \in P_{\delta}$ and $y \in Q_{\delta}\left(\subseteq Y_{\delta}[p]\right)$. Choose $\nu<\delta$ so that $a, x \in A_{\nu}$ and $\nu \notin E$. So $p^{n} \mid a+x+\Pi_{0 \nu}(y)$. But $\Pi_{0 \nu}(y) \in P_{\delta}$. (Here we use that $A$ is in standard form.) Since $A_{\delta}$ is pure there is $a^{\prime} \in A_{\delta}$ so that $p^{n} a^{\prime}=a+x+\Pi_{0 \nu}(y) \equiv a \bmod P_{\delta+1}$. Finally since $A_{\delta} / P_{\delta} \simeq\left(A_{\delta}+P_{\delta+1}\right) / P_{\delta+1}$ and $A_{\delta+1} / A_{\delta}+P_{\delta+1}$ are $\Sigma$-cyclic and $\left(A_{\delta}+P_{\delta+1}\right) / P_{\delta+1}$ is pure in $A_{\delta+1} / P_{\delta+1}, A_{\delta+1} / P_{\delta+1}$ is $\Sigma$-cyclic.

As will be proved, it is consistent that every $\omega_{1}$-separable group of cardinality $\omega_{1}$ can be put in standard form. So under some set-theoretic assumptions Theorem 2.2 
characterizes the $\omega_{1}$-separable groups of cardinality $\omega_{1}$ which are $p^{\omega+1}$-projective. By a theorem of Fuchs [F2] any $p^{\omega+1}$-projective group of final rank $\omega_{1}$ has a $\Sigma$ cyclic direct summand of final rank $\omega_{1}$. (The final rank of $G$ is $\lim _{n} \operatorname{rank}\left(G / p^{n} G\right)$.) Hence the following theorem shows that the characterization fails if $\diamond$ holds.

In the course of proving the next theorem we will need a particular pure projective resolution of a group of length $\omega+1$. We summarize the construction as a lemma.

2.3 LEMMA. Suppose $m_{n}(n<\omega)$ is an increasing sequence of natural numbers where $m_{0}=1$. Let $G$ be the $\bigoplus\left\langle g_{n}\right\rangle \oplus \bigoplus_{1 \leq n}\left\langle h_{n}\right\rangle$ where the order of $g_{n}$ is $p^{m_{n}}$ and the order of $h_{n}$ is $p^{m_{n}-1}$. For convenience we let $h_{0}=0$. For $n<\omega$, let $k_{n}=h_{n}-g_{n}-p^{m_{n+1}-m_{n}} g_{n+1}$. Let $K$ be the subgroup of $G$ generated by the $k_{n}$ 's.

(1) $p^{\omega}(G / K)=\left\langle g_{0}+K\right\rangle$ a cyclic group of order $p$.

(2) For all $j$ let $K_{j}$ be the subgroup generated by $\left\{k_{n}: n<j\right\}$. Then $\left\{k_{n}: n<j\right\}$ is a basis for $K_{j}$.

(3) For each $n$, the order of $k_{n}$ is $p^{m_{n}}$.

(4) For each $j$, let $H_{j}$ be the subgroup generated by $\left\{h_{n}: n<\omega\right\} \cup\left\{g_{n}: n \geq j\right\}$. Then $G=K_{j} \oplus H_{j}$.

(5) Finally for all $j$, ht $\left(g_{0}+K_{j}\right)$ in $G / K_{j}$ is $m_{j}$.

The proof of this lemma is a straightforward verification.

2.4 THEOREM. Assume $\diamond$ holds. There is an $\omega_{1}$-separable p-group A of cardinality $\omega_{1}$, final rank $\omega_{1}$ which is not the direct sum of two groups of final rank $\omega_{1}$. Further $A$ has an $\omega_{1}$-filtration $\left(A_{\alpha}: \alpha<\omega_{1}\right)$ such that for all $\alpha, A_{\alpha+1} / A_{\alpha}$ is $p^{\omega+1}$-projective.

PROOF. The proof we present here is a modification of Theorem 3.5 in [E3] which itself is a modification of [EM1]. To simplify the proof we will take $E$ a stationary costationary subset of $\operatorname{Lim}\left(\omega_{1}\right)$ such that $\diamond(E)$ holds. We will construct inductively $A_{\alpha}$ a group structure on $\alpha \times \omega$ and $B_{\nu \alpha}, \nu \leq \alpha, \nu \notin E$, so that each $A_{\alpha}$ is $\Sigma$-cyclic and $\left(B_{\nu \alpha}: \nu \leq \alpha, \nu \notin E\right)$ is a system of complementary summands. If $\alpha \notin E$, then $A_{\alpha+1} \simeq A_{\alpha} \oplus \bigoplus_{n \in \omega} Z\left(p^{n}\right)$ and the $B_{\nu \alpha+1}$ 's are defined in the obvious manner. At limit ordinals we take unions.

Using $\diamond(E)$ we choose $\left(\left(Y_{\delta}^{0}, Y_{\delta}^{1}\right): \delta \in E\right)$ so that for all $Y^{0}, Y^{1} \subseteq \omega_{1} \times \omega$ $\left\{\delta: Y^{0} \cap \delta \times \omega=Y_{\delta}^{0}\right.$ and $\left.Y^{\prime} \cap \delta \times \omega=Y_{\delta}^{1}\right\}$ is stationary. The key case occurs when

there is $\left(\alpha_{n}: n \in \omega\right)$ an increasing sequence of ordinals outside $E$ with limit $\delta$ so that for all $n, A_{\alpha_{n}}=Y_{\delta}^{0} \cap A_{\alpha_{n}} \oplus Y_{\delta}^{1} \cap A_{\alpha_{n}}$ and the final rank of $Y_{\delta}^{i} \cap A_{\alpha_{n+1}} / Y_{\delta}^{i} \cap A_{\alpha_{n}}$ is $\omega(i=0,1)$.

In this case since $Y_{\delta}^{i} \cap A_{\alpha_{n+1}} / Y_{\delta}^{i} \cap A_{\alpha_{n}}$ is $\Sigma$-cyclic we can choose $c_{n}^{i} \in Y_{\delta}^{i} \cap A_{\alpha_{n+1}}$ so that $c_{n}^{i}+Y_{\delta}^{i} \cap A_{\alpha_{n}}$ generates a pure subgroup and is of the same order as $c_{n}^{i}$. Further we can assume if $m<n$ then the order of $c_{m}^{i}$ is less than the order of $c_{n}^{j}(i, j=0,1)$. For all $n$ let $c_{n}=c_{n}^{0}$ if $n$ is even and $c_{n}^{1}$ otherwise. Let $m_{n}$ be the order of $c_{n}$. For all $n \neq 0$, let $b_{n}$ be the (unique) element of $B_{\alpha_{n} \alpha_{n+1}}$ so that $c_{n}-b_{n} \in A_{\alpha_{n}}$. Note that the order of $b_{n}$ is the same as that of $c_{n}$ and $b_{n}$ generates a pure subgroup of $B_{\alpha_{n} \alpha_{n+1}}$. Choose $b_{0} \in B_{\alpha_{0} \alpha_{1}}$ so that the order of $b_{0}$ is $p$.

Let $G, K, g_{n}, h_{n}, k_{n}$ be as in Lemma 2.3 relative to the sequence $m_{n}$. Define $A_{\delta+1} \cong A_{\delta}+G /\left\langle\left(b_{n},-k_{n}\right): n<\omega\right\rangle$. (We add $G$ to $A_{\delta}$ but identify $b_{n}$ and $k_{n}$.) For all $n$ let $B_{\alpha_{n} \delta+1}$ be the subgroup generated by $B_{\alpha_{n} \delta}$ together with $H_{n}$. As in [M1] we can verify that this induces a definition of the $B_{\nu \delta+1}$ 's. 
At other ordinals in $E$ we just choose the $b_{n}$ 's in any allowable manner.

Let $A=\bigcup A_{\alpha}$. The construction of the $B_{\nu \alpha}$ 's guarantee that $A$ is $\omega_{1}$-separable. So it remains to see that $A$ is not the direct sum of two groups of final rank $\omega_{1}$. Suppose $A=Y^{0} \oplus Y^{1}$ is a counterexample. Then as in [EM1] there is an ordinal $\delta \in E$ so that $Y^{0} \cap \delta \times \omega=Y_{\delta}^{0}, Y^{1} \cap \delta \times \omega=Y_{\delta}^{1}$ and $(*)$ holds at $\delta$.

By the construction $p^{\omega}\left(A / A_{\delta}\right) \simeq Z(p)$. So either $g_{0}+A_{\delta} \in Y^{0}+A_{\delta}$ or $g_{0}+$ $A_{\delta} \in Y^{1}+A_{\delta}$. Without loss of generality we can assume $g_{0}+a \in Y^{0}$ where $a \in A_{\delta}$. Choose $n$ odd so that $a \in A_{\alpha_{n}}$. By construction $\operatorname{ht}\left(g_{0}+A_{\alpha_{n}}\right)=m_{n}$ and ht $\left(g_{0}+\left\langle A_{\alpha_{n}} \cup\left\{b_{n}\right\}\right\rangle\right)=m_{n+1}$. But $\left\langle A_{\alpha_{n}} \cup\left\{b_{n}\right\}\right\rangle=\left\langle A_{\alpha_{n}} \cup\left\{c_{n}\right\}\right\rangle$. Also $c_{n} \in Y^{1}$. So ht $\left(g_{0}+\left\langle A_{\alpha_{n}} \cup\left\{c_{n}\right\}\right\rangle\right)=\operatorname{ht}\left(g_{0}+A_{\alpha_{n}}\right)$, a contradiction. (A small point is if $g_{0}+A_{\delta} \in Y+A_{\delta}$ we must take $n \geq 2$.)

The construction in the theorem above can be generalized to get $\Gamma(A)=1$ and any specified countable quotients.

Many of the constructions of pathological $\omega_{1}$-separable groups assuming some set theoretic axioms can be modified to constructions of groups in standard form. For example we can prove the following theorems. (We cite the papers where the results, without the requirement that the groups are in standard form, were proved.)

2.5 ThEOREM. (1) [E3, Theorem 3.5]. Assume $\diamond(E)$ for some stationary subset $E \subseteq \lim \left(\omega_{1}\right)$. Then there is an $\omega_{1}$-separable $\omega_{1}$-free group $A$ in standard form such that $\Gamma(A)=\tilde{E}$ and $A$ is not the direct sum of two uncountable groups.

(2) (Mekler, unpublished) In (1) A can also be taken to be "almost endo-rigid" i.e.. any endomorphism is, modulo a countable summand, multiplication by an integer.

(3) [EM2, Theorem 1.3]. Assume $\diamond(E)$ holds for some stationary subset $E \subseteq$ $\lim \left(\omega_{1}\right)$. Let $R$ be a ring whose additive group is the completion of a free $p$-adic module of countable rank. Then there is an $\omega_{1}$-separable $p$-group in standard form $A$ of final rank $\omega_{1}$ such that the endomorphism ring of $A$ is a split extension of $R$ by $E_{s}^{\omega}(A)$. (Here $E_{s}^{\omega}(A)$ are the unavoidable endomorphisms any $\omega_{1}$-separable p-group must have.)

PROOF. We shall only sketch the changes to the proof in [E3] necessary to prove the stronger theorem stated here. The group $A$ is constructed with $\omega_{1}$ as the underlying set. So we can use $\diamond(E)$ to predict pairs $\left\{\left(Y_{\delta}, Y_{\delta}^{\prime}\right): \delta \in E\right\}$ so thät if $Y, Y^{\prime} \subseteq A$ there is some $\delta$ such that $Y \cap A_{\delta}=Y_{\delta}$ and $Y^{\prime} \cap A_{\delta}=Y_{\delta}^{\prime}$. We construct $\left(A_{\alpha}: \alpha \in \omega_{1}\right)$ an $\omega_{1}$-filtration of $A,\left(B_{\alpha \nu}: \alpha \notin E, \alpha<\nu\right)$ a system of complementary summands, and $\left(Y_{\delta}: \delta \in E\right)$ witnessing that $A$ is in standard form by induction on $\alpha<\omega_{1}$. Further the construction will satisfy the additional condition:

for all $x$ and $n<\omega$ there is a finite set of ordinals $S$ so that $2^{n} \mid x-\sum_{\alpha \in S} \Pi_{\alpha \alpha+1}(x)$.

The only novel case in the induction occurs when

$\delta \in E, Y_{\delta}$ and $Y_{\delta}^{\prime}$ are subgroups of $A_{\delta}$ there is a ladder $\eta_{\delta}$ on $\delta$ such that for all $n \in \omega$, letting $\tau_{n}=\eta_{\delta}(n)$ :

$A_{\tau_{n}}=\left(Y_{\delta} \cap A_{\tau_{n}}\right)+\left(Y_{\delta}^{\prime} \cap A_{\tau_{n}}\right)$;

$Y_{\delta} \cap A_{\tau+1} / Y_{\delta} \cap A_{\tau_{n}}$ has a summand $\cong \mathbf{Z}$; and

$Y_{\delta}^{\prime} \cap A_{\tau_{n+1}} / Y_{\delta}^{\prime} \cap A_{\tau_{n}}$ has a summand $\cong \mathbf{Z}$. 
Now Eklof shows for all $n \in \omega$ there are $c_{n} \in Y_{\delta} \cap A_{\tau_{2 n+2}}$ and $c_{n}^{\prime} \in Y_{\delta}^{\prime} \cap A_{\tau_{2 n+2}}$ such that $c_{n}+c_{n}^{\prime} \in B_{\tau_{2 n+1}, \tau_{2 n+2}}$ and $c_{n}$ (resp. $c_{n}^{\prime}$ ) is of height 1 modulo $Y_{\delta} \cap A_{\tau_{2 n+1}}$ (resp. $Y_{\delta}^{\prime} \cap A_{\tau_{2 n+1}}$. By our inductive hypothesis we can choose a finite set of ordinals $S$ such that if $\alpha \in S$, then $\alpha \notin E$ and $2^{n+1} \mid c_{n}+c_{n}^{\prime}-\sum_{\alpha \in S} \Pi_{\alpha \alpha+1}\left(c_{n}+c_{n}^{\prime}\right)$. Let $b_{n}=\sum_{\alpha \in S} \Pi_{\alpha \alpha+1}\left(c_{n}+c_{n}^{\prime}\right)$. Let

$$
z_{n}=\frac{y_{\delta}-\sum_{i=0}^{n} 2^{i} b_{i}}{2^{n+1}} \text { and } A_{\delta+1}=\left\langle A_{\delta} \cup\left\{z_{n}: n \in \omega\right\}\right\rangle .
$$

As usual we can let $B_{\tau_{2 n}+1 \delta+1}=\left\langle B_{\tau_{2 n}+1 \delta} \cup\left\{z_{m}: m>n\right\}\right\rangle$ and $Y_{\delta}=\left\{z_{m}: m \in \omega\right\}$.

In order to see that $A$ is the desired group, note first that $2^{n+1} \mid y_{\delta}-\left(c_{n}+c_{n}^{\prime}\right)$ modulo $A_{\tau_{2 n}}$. Then just as in [E3] we can show that $A$ is not the direct sum of two uncountable groups.

REMARK. In particular Theorem 2.5.3 says that there is an $\omega_{1}$-separable $p$-group in standard form without a $\Sigma$-cyclic direct summand of final rank $\omega_{1}$. Because of the result of Fuchs quoted before Theorem 2.3 (i.e. that any $p^{\omega+1}$-projective group of final rank $\omega_{1}$ has $\Sigma$-cyclic direct summand of final rank $\left.\omega_{1}\right)$, there may seem to be an incompatibility between the claims in Theorem 2.5.3 and Theorem 2.2. (Certainly it caused the author to worry.) In the proof of 2.5.3, $A_{\delta+1}$ is contained in the torsion completion of $A_{\delta}$. So Theorem 2.2 does not apply. However Theorem 2.2 shows that in Theorem 2.5.3 we cannot have arbitrary quotients.

Although being in standard form has few consequences without some additional set theoretic hypothesis the situation changes if we assume $\mathrm{MA}+\neg \mathrm{CH}$. Since we will only need one consequence of $\mathrm{MA}+\neg \mathrm{CH}$, rather than reviewing this axiom we will just state the result we will need.

DEFinition. Suppose $\left(\eta_{\delta}: \delta \in E\right)$ is a ladder system on $E \subseteq \operatorname{Lim}\left(\omega_{1}\right)$. A collection of functions $\left(c_{\delta}: \delta \in E\right)$ where $c_{\delta}: \omega \rightarrow \omega_{1}$ is called a coloring of $\left(\eta_{\delta}: \delta \in\right.$ $E)$. A function $f: \omega_{1} \rightarrow \omega_{1}$ uniformizes $\left(c_{\delta}: \delta \in E\right)$ if for all $\delta \in E$ there is $n_{\delta}$ such that for all $m \geq n_{\delta} f\left(\eta_{\delta}(m)\right)=c_{\delta}(m)$.

2.6 THEOREM ([DS] or see [M4] for another proof). Assume MA $+\neg \mathrm{CH}$ and $\left(\eta_{\delta}: \delta \in E\right)$ is a ladder system on $E \subseteq \operatorname{Lim}\left(\omega_{1}\right)$. If $\left(c_{\delta}: \delta \in E\right)$ is a coloring of $\left(\eta_{\delta}: \delta \in E\right)$ and for all $\delta \in E$ and $n<\omega c_{\delta}(n) \leq \eta_{\delta}(n)$, then there is $f: \omega_{1} \rightarrow \omega_{1}$ which uniformizes the coloring.

Following Eklof we will define a Fuchs 5 group $A$ of cardinality $\omega_{1}$ to have the decomposition property if whenever $\Gamma(A)=\tilde{E}$ and $E=\bigcup_{i<\omega_{1}} E_{i}$ (disjoint) and for all $\delta \in E_{i}, \delta>i$ then $A=\bigoplus_{i<\omega} A^{i}$ where $\Gamma\left(A^{i}\right)=\tilde{E}_{i}$. In [E3] it is shown that assuming $\mathrm{MA}+\neg \mathrm{CH}$ and a decomposition property of stationary sets that any $\omega_{1}$-separable $\omega_{1}$-free group of cardinality $\omega_{1}$ has the decomposition property. (This is not the statement of Theorem 2.10 .2 of [E3], but is the content of the proof.) In [EM2] we generalized the methods of [E3] to $\omega_{1}$-separable $p$-groups of cardinality $\omega_{1}$. However the best result we were able to prove there was that, assuming $\mathrm{MA}+\neg \mathrm{CH}$, any such group is isomorphic to the direct sum of itself together with a $\Sigma$-cyclic group of final rank $\omega_{1}$. The following result together with the results in the next section, generalize in the context of PFA (or $\mathrm{PFA}^{-}$) the results discussed above.

2.7 THEOREM. Assume MA $+\neg \mathrm{CH}$. Suppose $A$ is a Fuchs 5 group in standard form. Then $A$ has the decomposition property. 
PROOF. Let $\left(A_{\delta}: \alpha<\omega_{1}\right),\left(B_{\alpha \nu}: \alpha \notin E, \alpha<\nu\right)$ and $\left(Y_{\delta}: \delta \in E\right)$ witness that $A$ is in standard form. We can assume that for all $\delta \in E$ and $\alpha<\delta, \alpha \in E$ $\left\{y \in Y_{\delta}: \Pi_{0 \alpha}(y) \neq 0\right\}$ is finite. Hence for $\delta \in E,\left\{\alpha<\delta: \Pi_{\alpha \alpha+1}(y) \neq 0\right.$, some $\left.y \in Y_{\delta}\right\}$ is of order type $\omega$. Let $\eta_{\delta}$ be the ladder enumerating this set. So we have a ladder system $\left(\eta_{\delta}: \delta \in E\right)$.

Suppose now that $E=\bigcup_{i<\omega_{1}} E_{i}$ (disjoint) where for all $\delta \in E_{i}, \delta>i$. We define a coloring $\left(c_{\delta}: \delta \in E\right)$ by

$$
c_{\delta}(n)= \begin{cases}i & \text { iff } \delta \in E_{i} \text { and } \eta_{\delta}(n)>i \\ 0 & \text { otherwise }\end{cases}
$$

Let $f: \omega_{1} \rightarrow \omega_{1}$ uniformize $\left(c_{\delta}: \delta \in E\right)$. For $i<\omega_{1}$, define $B^{i}=\bigoplus B_{\alpha \alpha+1}(\alpha \notin E$ and $f(\alpha)=i)$. For $i<\omega_{1}$ and $\delta \in E_{i}$ define $Y_{\delta}^{\prime}=\left\{y-\Pi_{0 k_{\delta}}(y): y \in Y_{\delta}\right\}$. (Here $\left(n_{\delta}: \delta \in E\right)$ is a sequence of natural numbers which witness that $f$ uniformizes $\left(c_{\delta}: \delta \in E\right)$ and $k_{\delta}=\max \left\{\eta_{\delta}\left(n_{\delta}\right), i+1\right\}$.) Now define $A^{i}=\left\langle B^{i} \cup\left\{Y_{\delta}^{\prime}: \delta \in E_{i}\right\}\right\rangle$. Since for all $\delta, i$ and $y \in Y_{\delta}, \Pi_{0 \eta_{\delta}\left(n_{\delta}\right)}(y)=\sum_{\alpha \in S} \Pi_{\alpha \alpha+1}(y)$ for some finite set $S \subseteq \omega_{1} \backslash E, A=\sum_{i<\omega_{1}} A^{i}$. It is also easy using the definition of the $\left(\eta_{\delta}: \delta \in E\right)$ to see that $A=\bigoplus_{i<\omega_{1}} A^{i}$.

We can now partially answer the question, "can all weak Fuchs 5 groups be put in standard form?" The answer assuming some set theory is no.

2.8 THEOREM. It is consistent with $\mathrm{MA}+\neg \mathrm{CH}$ that there is an $\omega_{1}$-separable $\omega_{1}$-free group of cardinality $\omega_{1}$ which cannot be put in standard form. Alternately if we assume $\diamond(E)$ holds for some stationary $E \subseteq \operatorname{Lim}\left(\omega_{1}\right)$, then there is an $\omega_{1}$ separable $\omega_{1}$-free group of cardinality $\omega_{1}, A$ which cannot be put in standard form and $\Gamma(A)=\tilde{E}$.

PROOF. In [E3, Theorem 2.10(1)], assuming $\diamond(E)$, an $\omega_{1}$-separable $\omega_{1}$-free group of cardinality $\omega_{1}$ is constructed with $\Gamma(A)=\tilde{E}$. This group has the property that if $\mathrm{MA}+\neg \mathrm{CH}$ is forced true by a c.c.c poset then $A$ does not have the decomposition property. So neither in the ground model nor in the forcing extension can $A$ be put in standard form. Theorems 2.2 and 2.4 show that $\diamond(E)$ implies that there is an $\omega_{1}$-separable $p$-group of cardinality $\omega_{1}$ which cannot be put in standard form with $\Gamma(A)=\tilde{E}$.

REMARK. The proof of the theorem above has some disadvantages. The groups Eklof constructs are of type $R$ (i.e. for $\delta \in E A_{\delta+1} / A_{\delta} \simeq\{a / b \in \mathbf{Q}: b$ is square free $\})$. Also his group possesses a system of complementary summands. There are a few natural questions.

QUESTION. Is it consistent that any $\omega_{1}$-separable $\omega_{1}$-free group of cardinality $\omega_{1}$ is quotient equivalent to one which cannot be put in standard form (i.e. can the $A_{\delta+1} / A_{\delta}$ be arbitrary)? Is it consistent that there is an $\omega_{1}$-separable $\omega_{1}$-free group of cardinality $\omega_{1}$ which has no system of complementary summands?

3. PFA and standard form. In this section we will show it is consistent that most weak Fuchs 5 groups can be put in standard form. Our results will be consequences of the proper forcing axiom, PFA. We will review the definitions needed to understand this axiom (but again we recommend [M3]).

Definition. If $A$ is a set $\mathcal{P}_{\omega_{1}}(A)$ denotes the set of all countable subsets of $A$. A subset $C \subseteq P_{\omega_{1}}(A)$ is called a $c u b$ if it is closed under the union of countable 
chains and if for $X \in \mathcal{P}_{\omega_{1}}(A)$ there is $Y \in C$ so that $X \subseteq Y$. If $\kappa$ is a cardinal then by $H(\kappa)$ we denote the sets of hereditary cardinality $<\kappa$ (i.e. $H(\kappa)$ is the unique set such that $X \in H(\kappa)$ iff $|X|<\kappa$ and for all $Y \in X, Y \in H(\kappa))$. If $A$ is a set we say $\kappa$ is large enough for $A$ if $A, P(A) \in H(\kappa)$. Suppose $\mathbf{P}$ is a partially ordered set (a poset). All posets are assumed to have a least element 0 . A subset $D \subseteq \mathbf{P}$ is dense if for all $p \in \mathbf{P}$ there is $q \in D$ so that $p \leq q$ and for all $p \in D, p \leq q$ implies $q \in D$. Elements $p, q \in \mathbf{P}$ are compatible if there is $r$ such that $p, q \leq r$.

Suppose now $N \prec(H(\kappa), \in, \mathbf{P})$ where $\kappa$ is large enough for $\mathbf{P}$. A $q \in \mathbf{P}$ is $N$ generic if for all $r \geq q$ and $D \in N$ a dense subset of $\mathbf{P}$ there is $s \in D \cap N$ such that $r$ and $s$ are compatible. A poset $\mathbf{P}$ is proper iff for any $\kappa$ large enough for $\mathbf{P}$ there is a cub set of countable $N \prec(H(\kappa), \in, \mathbf{P})$ so that all $p \in N \cap \mathbf{P}$ there is $p \leq q$ which

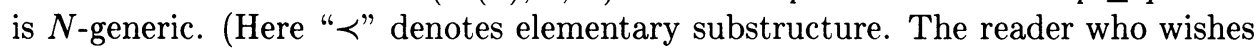
to ignore this notion can just assume $N$ has all appropriate closure properties. Also it is equivalent to require the condition above for all such $N$.)

DEFINITION. $\operatorname{PFA}(\lambda)$ : If $\mathbf{P}$ is a proper poset of cardinality $\leq \lambda$ and $D$ is a family of $\omega_{1}$ dense subsets of $\mathbf{P}$, then there is a $D$-generic subset of $\mathbf{P}$ (i.e. there is a directed subset $G$ of $\mathbf{P}$ such that $G \cap D \neq \varnothing$ for all $D \in D)$.

PFA: for all cardinals $\lambda, \operatorname{PFA}(\lambda)$.

Shelah [S2] has shown that PFA is consistent with ZFC, assuming the consistency of the existence of a certain large cardinal. For our purposes $\operatorname{PFA}\left(2^{\aleph_{0}}\right)$ will suffice. One also needs a large cardinal to show the consistency of $\operatorname{PFA}\left(2^{\aleph_{0}}\right)$. However all our results actually follow from an axiom, $\mathrm{PFA}^{-}$, we formulated in [M3]. This axiom can be shown to be relatively consistent with ZFC (without any large cardinal assumptions). Unfortunately it has a complicated statement. So we will state that our results are consequences of $\mathrm{PFA}^{-}$but prove them assuming $\operatorname{PFA}\left(2^{\aleph_{0}}\right)$. (It should be noted that our contribution in showing the consistency of $\mathrm{PFA}^{-}$was restricted to explicitly stating the axiom. Everything else is implicit in [S2]. In this paper we will not bother stating $\mathrm{PFA}^{-}$.)

3.1 TheOREM. Assume $\mathrm{PFA}^{-}$. Suppose $A$ is a weak Fuchs 5 group of cardinality $\omega_{1}$; then $A$ can be put in standard form provided either that

(I) $\Gamma(A) \neq 1$ or

(II) every countable subgroup of $A$ is a direct sum of cyclic groups.

PROOF. To begin choose an $\omega_{1}$-filtration $\left(A_{\alpha}: \alpha<\omega_{1}\right)$ of $A$ so that for each $\alpha, A_{\alpha+1}$ is $\omega_{1}$-pure. Let $E=\left\{\alpha: A_{\alpha}\right.$ is not $\omega_{1}$-pure $\}$. We want to find a cub $C \subseteq \omega_{1}$, a system of complementary summands $\left\{B_{\nu \tau}: \nu, \tau \in C, \nu \notin E\right\}$ and $\left\{Y_{\delta}: \delta \in E \cap C\right\}$ such that:

(1) if $\delta \in C \cap E$, then $\delta+1 \in C$;

(2) if $\sigma \in C$ then $\sigma^{+} \notin E$ (where $\sigma^{+}$is the successor of $\sigma$ in $C$ );

(3) if $\delta \in C \cap E$ then $A_{\delta+1}=\left\langle A_{\delta} \cup Y_{\delta}\right\rangle$;

(4) if $\delta \in C \cap E$, then for all $\nu, \tau \in C \cap \delta$ with $\nu, \tau \notin E$ and $y \in Y_{\delta}$ there is $S$ a finite subset of $C \cap \delta$ such that $\Pi_{\nu \tau}(y)=\sum_{\sigma \in S} \Pi_{\sigma \sigma^{+}}(y)$.

We let our poset $\mathbf{P}$ be the partial order whose elements are countable approximations to the desired sets. More exactly an element of $\mathbf{P}$ is a triple $(C, B, \mathcal{Y})$ where $C$ is a countable closed subset of $\omega_{1}$ with $\sup C \notin E, B=\left(B_{\nu \tau}: \nu, \tau \in C, \nu \notin E\right)$ is a system of complementary summands, and $\mathcal{Y}=\left\{Y_{\delta}: \delta \in C \cap E\right\}$ and $C, B, \mathcal{Y}$ satisfy 
(1)-(4). Order $\mathbf{P}$ by letting $(C, B, y) \leq\left(C^{\prime}, B^{\prime}, Y^{\prime}\right)$ iff $C^{\prime}$ is an end-extension of $C$, $B \subseteq B^{\prime}$ and $\mathcal{Y} \subseteq \mathcal{Y}^{\prime}$.

For $\alpha<\omega_{1}$ let $D_{\alpha}=\{(C, B, y): \alpha \leq \sup C\}$. We claim that each $D_{\alpha}$ is dense. Consider any $(C, B, \mathcal{Y}) \in \mathbf{P}$ and assume $\beta=\sup C<\alpha$. Choose $B_{\beta \alpha+1}$ such that $A_{\beta} \oplus B_{\beta \alpha+1}=A_{\alpha+1}$. For $\nu \in C \cap \beta$ and $\nu \notin E$, let $B_{\nu \alpha+1}=B_{\nu \beta} \oplus B_{\beta \alpha+1}$. Let $C^{\prime}=C \cup\{\alpha+1\}$ and $B^{\prime}=B \cup\left\{B_{\nu \alpha+1}: \nu \in C, \nu \notin E\right\}$. Then $(C, B, \mathcal{Y})<$ $\left(C^{\prime}, B^{\prime}, Y\right) \in D_{\alpha}$. Assume for the moment that $\mathbf{P}$ is proper. If $G$ is a directed subset of $\mathbf{P}$ which meets each $D_{\alpha}$, then $(\bigcup C, \cup B, \cup \mathcal{Y})$, where all unions are taken over $(C, B, \mathcal{Y}) \in G$, are the desired sets. It remains to see that $\mathbf{P}$ is proper. We consider the cases (I) and (II) separately.

Assume (I). Our proof is modelled on the proof in [M2]. We consider a countable $N \prec(H(\kappa), \in, \mathbf{P})$ and let $\alpha=N \cap \omega_{1}$ (note: $N \cap \omega_{1}$ is always a countable ordinal). We can further assume $N=\bigcup_{n<\omega} N_{n}$, where for all $n N_{n} \prec N_{n+1} \prec(H(\kappa), \in, \mathbf{P})$, $\alpha_{n}<\alpha_{n+1}$, and $\alpha_{n} \notin E$. Here $\alpha_{n}=N_{n} \cap \omega_{1}$. We also assume that $\left(A_{\nu}: \nu<\omega_{1}\right) \in$ $N_{0}$. So for all $n$ if $\beta<\alpha_{n}$, then $A_{\beta} \in N_{n}$. There are two cases to consider: either $\alpha \in E$ or $\alpha \notin E$. The case where $\alpha \notin E$ is substantially easier so we assume that $\alpha \in E$.

Consider $\left(C_{0}, B_{0}, Y_{0}\right) \in \mathbf{P} \cap N$. Without loss of generality we can assume $\left(C_{0}, B_{0}, Y_{0}\right) \in N_{0}$. Enumerate the dense subsets of $\mathbf{P}$ in $N$ as $\left(D_{n}: n<\omega\right)$ so that $D_{n} \in N_{n}$. Next choose $\left(H_{n}: n<\omega\right)$ and $\left(K_{n}: n<\omega\right)$ so that $A_{\alpha_{n}} \oplus H_{n}=A_{\alpha+1}$ and $H_{n}=K_{n} \oplus H_{n+1}$. (Such a sequence can be chosen inductively by first choosing $H_{0}$, letting $K_{0}=H_{0} \cap A_{\alpha_{1}}$ then choosing $H_{1}$ so that $K_{0} \oplus H_{1}=H_{0}$, etc.) Let $\psi_{n}$ be the projection of $A_{\alpha+1}$ on $K_{n}$ along $A_{\alpha_{n}} \oplus H_{n+1}$ and let $\psi_{-1}$ be the projection of $A_{\alpha+1}$ on $A_{\alpha_{0}}$ along $H_{0}$. Choose $Y_{\alpha}$ so that $A_{\alpha+1}=\left\langle A_{\alpha} \cup Y_{\alpha}\right\rangle$, for all $y \in Y_{\alpha}, \psi_{-1}(y)=0$, and for all $n,\left\{y \in Y_{\alpha}: \psi_{n}(y) \neq 0\right\}$ is finite.

We will now define a sequence of elements of $\mathbf{P},\left(C_{0}, B_{0}, Y_{0}\right)<\left(C_{0}^{\prime}, B_{0}^{\prime}, Y_{0}^{\prime}\right)<$ $\left(C_{1}, B_{1}, \mathcal{Y}_{1}\right)<\left(C_{1}^{\prime}, B_{1}^{\prime}, \mathcal{Y}_{1}^{\prime}\right)<\cdots$. We will let $\beta_{n}$ denote $\sup C_{n}$ and $\gamma_{n}$ denote $\sup C_{n}^{\prime}$. The sequence shall be chosen so that: $\left(C_{n}^{\prime}, B_{n}^{\prime}, \mathcal{Y}_{n}^{\prime}\right) \in N_{n} \cap D_{n}$; for all $n$, $\alpha_{n-1} \in C_{n}^{\prime}$; and for all $n$ and $y \in Y_{\alpha} \psi_{n}(y) \in B_{\alpha_{n} \alpha_{n}^{+}}$. Suppose for the moment we have defined such a sequence. We will finish the proof.

Let $C=\bigcup C_{n}, B=B_{n}$ and $y=\bigcup Y_{n}$. We will now extend $(C, B, Y)$ to a $\left(C^{\prime}, B^{\prime}, Y^{\prime}\right) \in \mathbf{P}$. (This clearly suffices, since if $\left(C^{\prime \prime}, B^{\prime \prime}, Y^{\prime \prime}\right) \geq\left(C^{\prime}, B^{\prime}, \mathcal{Y}^{\prime}\right)$ it is also $\geq\left(C_{n}, B_{n}, Y_{n}\right) \in D_{n} \cap N_{n}$.) Let $C^{\prime}=C \cup\{\alpha, \alpha+1\}$ and $\mathcal{Y}^{\prime}=\mathcal{Y} \cup\left\{Y_{\alpha}\right\}$. For $\nu \in$ $C \backslash E$, let $B_{\nu \alpha}=\bigcup_{\nu<\beta<\alpha} B_{\nu \beta}$. It remains to define $B_{\nu \alpha+1}$ for $\nu \in C \backslash E$. It suffices to define $B_{\alpha_{n} \alpha+1}$ for $n<\omega$. Let $B_{\alpha_{n} \alpha+1}=B_{\alpha_{n} \alpha} \cup\left\{y-\sum_{k<n} \psi_{k}(y): y \in Y_{\alpha}\right\}$. Then we let $B^{\prime}=B \cup\left\{B_{\nu \alpha}: \nu \in C \backslash E\right\} \cup\left\{B_{\nu \alpha+1}: \nu \in C \backslash E\right\}$.

The proof that $A_{\alpha+1}=A_{\alpha_{n}} \oplus B_{\alpha_{n} \alpha+1}$ is similar to that in [M2]. We repeat the argument here for the reader's convenience. Suppose $b+\sum_{i=1}^{k} a_{i} y_{i}^{\prime} \in A_{\alpha_{n}}$ where $b \in B_{\alpha_{n} \alpha}$ and each $y_{i}^{\prime}=y_{i}-\sum_{j<n} \psi_{j}(y)$ for some $y_{i} \in Y_{\alpha}$. Choose $m>n$ so that $b \in A_{\alpha_{m}}$. Let $\psi=\psi_{-1}+\psi_{0}+\cdots+\psi_{m-1}$. Then

$$
\begin{aligned}
b+\sum_{i=1}^{k} a_{i} y_{i}^{\prime} & =\psi\left(b+\sum_{i=1}^{k} a_{i} y_{i}^{\prime}\right) \quad\left(\text { since } \psi \text { is the identity on } A_{\alpha_{m}}\right) \\
& =b+\sum_{i=1}^{k} a_{i} \psi\left(y_{i}^{\prime}\right)=b+\sum_{i=1}^{k} \sum_{j=1}^{m-1} a_{i} \psi_{j}\left(y_{i}^{\prime}\right) .
\end{aligned}
$$


Further for all $i$,

$$
\sum_{j=1}^{m-1} \psi_{j}\left(y_{i}^{\prime}\right)=\sum_{j=n}^{m-1} \psi_{j}\left(y_{i}^{\prime}\right) \in B_{\alpha_{n} \alpha_{m}} .
$$

So $b+\sum_{i=1}^{k} a_{i} y_{i}^{\prime} \in B_{\alpha_{n} \alpha_{m}}$. Since $A_{\alpha_{n}} \cap B_{\alpha_{n} \alpha_{m}}=\{0\}$, this element must be 0 .

We now define the sequence. If $\left(C_{n}, B_{n}, Y_{n}\right) \in N_{n}$ has been defined, then choose $\left(C_{n}, B_{n}, Y_{n}\right)<\left(C_{n}^{\prime}, B_{n}^{\prime}, Y_{n}^{\prime}\right) \in N_{n} \cap D_{n}$. Now suppose $\left(C_{n}^{\prime}, B_{n}^{\prime}, Y_{n}^{\prime}\right)$ has been defined. Choose $B_{\gamma_{n} \alpha_{n}} \in N_{n+1}$ so that $A_{\gamma_{n} \alpha_{n}} \oplus B_{\gamma_{n} \alpha_{n}}=A_{\alpha_{n}}$. (Since such a subgroup of $A$ exists in $H(\kappa)$, one must exist in $N_{n+1}$.) Then choose $\beta_{n+1}$ so that $\alpha_{n}<\beta_{n+1}<$ $\alpha_{n+1}$ and $\left\{\psi_{n}(y): y \in Y_{\alpha}\right\} \in A_{\beta_{n}}$. Since $\left\{\psi_{n}(y): y \in Y_{\alpha}\right\}$ is finite and there is a group $B$ so that $A_{\alpha_{n}} \oplus B=A_{\beta_{n+1}}$ and $B \supseteq\left\{\psi_{n}(y): y \in Y_{\alpha}\right\}\left(K_{n} \cap A_{\beta_{n+1}}\right.$ works), there is $B_{\alpha_{n} \beta_{n+1}} \in N_{n+1}$ as above. Finally for $\nu \in C_{n}^{\prime} \backslash E$ let $B_{\nu \alpha_{n}}=B_{\nu \gamma_{n}} \oplus B_{\gamma_{n} \alpha_{n}}$ and $B_{\nu \beta_{n+1}}=B_{\nu \alpha_{n}} \oplus B_{\alpha_{n} \beta_{n+1}}$. (By convention let $B_{\nu \nu}=\{0\}$.) Define $C_{n+1}=$ $C_{n}^{\prime} \cup\left\{\alpha_{n}, \beta_{n+1}\right\}, B_{n+1}=B_{n} \cup\left\{B_{\nu \alpha_{n}}: \nu \in C_{n}^{\prime} \backslash E\right\} \cup\left\{B_{\nu \beta_{n+1}}: \nu \in C_{n}^{\prime} \backslash E\right.$ or $\left.\nu=\alpha_{n}\right\}$ and $y_{n+1}=y_{n}^{\prime}$.

Suppose now II holds. We can also assume $E=\lim \left(\omega_{1}\right)$ since otherwise we are in case I. The proof follows the same strategy as before, but we have to more clever in our choice of the $H_{n}$ 's and $K_{n}$ 's. We will need to use the following lemma which is a variant of a lemma we learned from L. Fuchs.

3.2 LEMMA. Suppose $G_{0} \subseteq G_{1} \subseteq G_{2}, G_{2}$ is a direct sum of cyclic groups, $G_{1}$ is a direct summand of $G_{2}, G_{0}=\bigcup_{i<\omega} R_{i}$ where for all $i R_{i} \subseteq R_{i+1}$ and $R_{i}$ is a direct summand of $G_{2}$. If $M$ is a finitely generated subgroup of $G_{2}$ and $G_{1} \cap M \subseteq G_{0}$, then there is $i$ and a finitely generated $M_{1}$ so that $M \subseteq R_{i}+M_{1}$ and for all $m \geq i$ there is $D \supseteq M_{1}$ with $R_{m} \oplus D=G_{2}$.

We will delay the proof of this lemma. We can assume we are considering $N=$ $\bigcup N_{n}, D_{n}(n<\omega),\left(C_{0}, B_{0}, Y_{0}\right), \alpha_{n}(n<\omega)$ which have the same properties as in case I, except $\alpha_{n} \in E$. We will inductively define an increasing sequence $m(n)(n<$ $\omega)$ of natural numbers, subgroups $K_{n}, H_{n}$ of $A_{\alpha+1},\left(C_{n}, B_{n}, \mathcal{Y}_{n}\right),\left(C_{n}^{\prime}, B_{n}^{\prime}, \mathcal{Y}_{n}^{\prime}\right) \in \mathbf{P}$, and $\left\{y_{i}^{n}: i<\omega\right\} \subseteq A_{\alpha+1}$. To begin choose $Y=\left\{y_{i}: i<\omega\right\}$ so that $A_{\alpha+1}=$ $\left\langle A_{\alpha} \cup Y\right\rangle$. Next let $m(0)=0$ and choose $H_{0}$ so that $A_{\alpha+1}=A_{\alpha_{0}+1}+H_{0}$. Let $\Pi_{-1}$ be the projection of $A_{\alpha+1}$ on $A_{\alpha_{0}+1}$ along $H_{0}$. For $i<\omega$ let $y_{i}^{0}=y_{i}-\Pi_{-1}\left(y_{i}\right)$. Choose $\left(C_{0}, B_{0}, Y_{0}\right)<\left(C_{0}^{\prime}, B_{0}^{\prime}, Y_{0}^{\prime}\right) \in N_{0} \cap D_{0}$. (As before we will let $\beta_{n}=\sup C_{n}$ and $\gamma_{n}=\sup C_{n}^{\prime}$.)

Next we will apply Lemma 3.2. Let $G_{2}=H_{0}$ and $M=\left\langle y_{0}^{0}\right\rangle$. Choose $m(0)<$ $m(1)$ so that $M \cap A_{\alpha}=M \cap A_{\alpha_{m(1)}}$. Let $G_{0}=A_{\alpha_{m(1)}} \cap H_{0}$ and $G_{1}=A_{\alpha_{m(1)}+1} \cap H_{0}$. Let $\tau_{i}(i<\omega)$ be a increasing sequence of successor ordinals $>\alpha_{0}+1$ with supremum $\alpha_{m(1)}$. Let $R_{i}=A_{\tau_{i}} \cap H_{0}$. Let $i$ and $M_{1}$ be as in the conclusion of Lemma 3.2. Choose a finite set $S \subseteq A_{\tau_{i}} \cap H_{0}$ so that $M \subseteq\left\langle S \cup M_{1}\right\rangle$. Now in $N_{\alpha_{m(1)}}$, choose first $B_{\gamma_{0} \alpha_{0}+1}$ and then choose $B_{\alpha_{0}+1} \tau_{i} \supseteq S$. Let $C_{1}=C^{\prime} \cup\left\{\alpha_{0}+1, \tau_{i}\right\}$, $Y_{1}=Y_{0}^{\prime}$, and let $B_{1}$ be the system of complementary summands determined by $B_{0}^{\prime} \cup\left\{B_{\alpha_{0}+1 \tau_{i}}, B_{\gamma_{0} \alpha_{0}+1}\right\}$. Next choose $\left(C_{1}, B_{1}, Y_{1}\right)<\left(C_{1}^{\prime}, B_{1}^{\prime}, Y_{1}^{\prime}\right) \in N_{m(1)} \cap D_{1}$. Next choose $W$ so that $W \supseteq M_{1}$ and $H_{0}=H_{0} \cap A_{\gamma_{1}} \oplus W$. Let $K^{\prime}=W \cap A_{\alpha_{m(1)}+1}$. Choose $H_{1}$ a complementary summand of $K^{\prime}$ in $W$. Let $T$ be a finite subset of $K^{\prime}$ so that $M_{1} \subseteq\left\langle T \cup H_{1}\right\rangle$. Now let $B_{\gamma_{1} \alpha_{m(1)}+1} \in N_{m(1)+1}$ be chosen so as to contain $T$. Finally let $K_{0}=H_{0} \cap A_{\gamma_{1}} \oplus K^{\prime}$. Note that with this choice of $K_{0}$ and $H_{1}$, $\Pi_{0}(M) \subseteq B_{\alpha_{0}+1 \beta_{1}}+B_{\gamma_{1} \alpha_{m(1)}+1}$ and $\beta_{1}=\left(\alpha_{0}+1\right)^{+}$and $\left(\gamma_{1}\right)^{+}=\alpha_{m(1)}+1$. (Here 
$\Pi_{0}$ is the projection on $K_{0}$ along $H_{1}$ and the equation $\left(\gamma_{1}\right)^{+}=\alpha_{m(1)}+1$ holds on $C_{2}$ which we are at liberty to define.) We then let $y_{i}^{1}=y_{i}^{0}-\Pi_{0}\left(y_{i}^{0}\right)(i<\omega)$.

In general for $n$ we repeat the construction above inside $H_{n}$ and let $M=\left\langle y_{i}^{n}: i \leq\right.$ $n\rangle$. To complete the construction we merely define $Y_{\alpha}=\left\{y_{n}^{n}: n<\omega\right\}$ and claim the rest of the proof is exactly as in case I.

We now give the promised proof of Lemma 3.2.

PROOF OF LEMMA 3.2. Since $M$ is finitely generated we can assume $G_{2}=$ $G_{1} \oplus L$ where $L$ is finitely generated. Let $\Pi: M \rightarrow L$ be the projection of $M$ into $L$ along $G_{1}$. Since $\operatorname{ker}(\Pi) \subseteq G_{1}, \operatorname{ker}(\Pi) \subseteq G_{0}$. Now let $c_{1}, \ldots, c_{k}$ be a set of independent generators of $\Pi(M)=L^{\prime}$. For each $j$ choose $g_{j} \in G_{1}$ so that $c_{j}+g_{j} \in M$. For any $j$ if there is $r_{j} \neq 0$ such that $r_{j} c_{j}=0$ and $r_{j} g_{j} \neq 0$, then $r_{j} g_{j} \in G_{0}$. Choose $r_{j}$ minimal and $g_{j}^{\prime} \in G_{0}$ so that $r_{j} g_{j}^{\prime}=r_{j} g_{j}$. If no such $r_{j}$ exists let $g_{j}^{\prime}=0$.

Define a map $\varphi: L^{\prime} \rightarrow G_{1}$ by $\varphi\left(c_{j}\right)=g_{j}-g_{j}^{\prime}$. We view $\varphi$ as a map to $G_{1} / R_{n}$ for all $n$. Choose $n_{1}, \psi, L^{\prime} \subseteq L^{\prime \prime} \subseteq L$ so that $\psi: L^{\prime \prime} \rightarrow G_{1} / R_{n_{1}}, \psi$ extends $\varphi$ and $L^{\prime \prime}$ is maximal. More exactly we assume that $L^{\prime \prime}+t(L) / t(L)$ is maximal in $L / t(L)$ and $L^{\prime \prime} \cap t(L)$ is of maximum cardinality. Now choose $i \geq n_{1}$ so that $\operatorname{ker}(\Pi) \subseteq R_{i}$, and for all $j, g_{j}^{\prime} \in R_{i}$. Since $R_{i}$ is a direct summand of $G_{1}$, we can assume $\psi$ is a map to a complementary summand of $R_{i}$ in $G_{1}$. Let $M_{1}=\left\{(\psi(c), c): c \in L^{\prime \prime}\right\}$. By the choice of $M_{1}, M \subseteq R_{i}+M_{1}$. To see that for any $m \geq i$ there is $D \supseteq M$ with $R_{m} \oplus D=G_{2}$ it is enough to check that $M_{1}$ is pure. ( $M_{1}$ is finitely generated.)

Consider any element $(\psi(c), c) \in M_{1}$ and suppose for some prime $p, p^{n} \mid(\psi(c), c)$ in $G_{1} / R_{m}$ but $p^{n} \nmid(\psi(c), c)$ in $M_{1}$. Choose $d$ so that $\langle d\rangle$ is pure in $L^{\prime \prime}$ and $c \in\langle d\rangle$. Assume $p^{l} k d=c$ where $\left(k, p^{l}\right)=1$. Fix $e \in L$ and $f \in G_{1} / R_{m}$ so that $p^{n}(f, e)=(\psi(c), c)$. Since $L$ and $G_{1} / R_{m}$ are $\Sigma$-cyclic, we can write $L$ as $L_{0} \oplus L_{p} \oplus L_{r}$ and $G_{1} / R_{m}$ as $H_{0} \oplus H_{p} \oplus H_{r}$ where $L_{0}, H_{0}$ are torsion free, $L_{p}, H_{p}$ are $p$-groups and $L_{r}, H_{r}$ are torsion groups with no elements of order $p$. We denote the obvious projections by the subscripts $0, p$ and $r$.

Choose integers $u$ and $v$ so that $k u \equiv 1 \bmod \left(\right.$ order of $\left.e_{p}\right)$ and $p^{n} v \equiv p^{l} \bmod$ (order of $d_{r}$ ). Let $g_{0}$ be the (unique) element of $L_{0}$ so that $k g_{0}=e_{0}$ and let $h_{0}$ be the element of $H_{0}$ so that $k h_{0}=f_{0}$. Let $g=g_{0}+u e_{p}+v d_{r}$ and $h=h_{0}+u f_{p}+v \psi(d)_{r}$. Note that $p^{n} k g=c$ and $p^{n} k h=\psi(c)$. Next choose $K$ so that $L^{\prime \prime}=\langle d\rangle \oplus K$. Let $L^{\prime \prime \prime}=\langle g\rangle \oplus K$. (Since $c \in\langle g\rangle,\langle g\rangle \cap K=0$.) Let $\psi^{\prime}: L^{\prime \prime \prime} \rightarrow G_{1} / R_{m}$ the unique màp extending $\psi \mid K$ such that $\psi^{\prime}(g)=h$. Now if $g_{0} \neq 0$ then $L^{\prime \prime \prime}+t(L) / t(L)$ strictly contains $L^{\prime \prime}+t(L) / t(L)$, contradicting the choice of $L^{\prime \prime}$. But if $g_{0}=0$, then $\left|L^{\prime \prime \prime} \cap t(L)\right|>\left|L^{\prime \prime} \cap t(L)\right|$. Again we have a contradiction.

3.3 Corollary (PFA $\left.{ }^{-}\right)$. Any weak Fuchs 5 group $A$ of cardinality $\omega_{1}$ is a Fuchs 5 group provided either that $\Gamma(A) \neq 1$ or $A$ is $\omega_{1}-\Sigma$-cyclic.

This result follows for $\omega_{1}$-free groups from Shelah's solution to the Whitehead problem ([S1] or see [M1] where this consequence was first observed). For $p$-groups it follows from Megibben's work on Crawley's problem [Meg1] and is specifically stated in [Meg2]. Later we will sketch a construction of an $\omega_{1}$-separable $\omega_{1}-\Sigma$ cyclic group which is not split. So our result even for $\omega_{1}$-separable $\omega_{1}-\Sigma$-cyclic groups is new. However the result for these groups could be proved by a standard $\mathrm{MA}+\neg \mathrm{CH}$ argument. The principal reason for giving this construction remains to illustrate the naturality of standard form. 
For weakly $\omega_{1}$-separable $\omega_{1}$-free groups of cardinality $>\omega_{1}$, Corollary 3.3 fails (in ZFC). Shelah has constructed (cf. [E2, Theorem 8.3]) a weakly $\omega_{1}$-separable $\omega_{1}$ free $A$ group of cardinality $2^{\omega_{1}}$ which is not a Whitehead group. So if $\mathbf{Z} \rightarrow B \rightarrow A$ is a nontrivial extension, then $B$ is weakly $\omega_{1}$-separable but not $\omega_{1}$-separable.

\section{Constructing a group in standard form.}

4.1 THEOREM. There is an $\omega_{1}$-separable $\omega_{1}-\Sigma$-cyclic group $A$ in standard form so that $A$ is not split (i.e. the torsion subgroup of $A$ is not a direct summand).

ProOF. First choose $G$ a countable group which is not split. Now let $K \rightarrow$ $F \rightarrow G$ be a pure projective resolution of $G$. That is $F$ and $K$ are $\Sigma$-cyclic and $K$ is pure in $F$. Since any finitely generated pure subgroup of $K$ is a direct summand of $F$ we can choose $K_{0} \subseteq K_{1} \cdots$ and $H_{0} \subseteq H_{1} \cdots$ so that $K=\bigoplus K_{n}$, for all $n F=\bigoplus_{m \leq n} K_{m} \oplus H_{n}$ and $H_{n}=K_{n} \oplus H_{n+1}$. By adding a $\Sigma$-cyclic group to all the groups we can assume each $K_{n}=\bigoplus_{n} \bigoplus_{p} \mathbf{Z}\left(p^{n}\right)^{(\omega)} \oplus \mathbf{Z}^{(\omega)}$. (Here $\mathbf{Z}\left(p^{n}\right)$ is the cyclic group of $p^{n}$ and the superscript $(\omega)$ indicates the direct sum of $\omega$ copies.)

Fix $E \subseteq \lim \left(\omega_{1}\right)$ a stationary set and $\left(\eta_{\delta}: \delta \in E\right)$ a ladder system. We define $\left(A_{\alpha}: \alpha<\omega_{1}\right),\left(B_{\alpha \nu}: \alpha<\nu, \alpha \in E\right)$ and $\left(Y_{\delta}: \delta \in E\right)$ by induction on $A$. For $\alpha \notin E$ choose $B_{\alpha \alpha+1} \cong \bigoplus_{n} \bigoplus_{p} \mathbf{Z}\left(p^{n}\right)^{(\omega)} \oplus \mathbf{Z}^{(\omega)}$ and let $A_{\alpha+1}=A_{\alpha}+B_{\alpha \alpha+1}$. Then let $B_{\tau \alpha+1}=\beta_{\tau \alpha} \oplus B_{\alpha \alpha+1}$, where this makes sense. At limit ordinals there is no choice. For $\delta \in E$, choose $Y_{\delta} \supseteq \bigoplus B_{\eta_{\delta}(n) \eta_{\delta}(n)+1}$, so that $F \cong Y_{\delta}$ via an isomorphism $\psi$ which takes $K_{n}$ to $B_{\eta_{\delta}(n)} \eta_{\delta}(n)+1$. Then let

$$
A_{\alpha+1}=Y_{\delta} \oplus B_{0 \eta_{\delta}(0)} \oplus \bigoplus B_{\eta_{\delta}(n)+1 n_{\delta}(n+1)} .
$$

Finally define $B_{\eta_{\delta}(n) \alpha+1}=\bigoplus_{m>n} B_{\eta_{\delta}(m)+1} \eta_{\delta}(m+1)+\psi\left(H_{n}\right)$. As usual $A=\bigcup A_{\alpha}$.

Suppose $A$ is split, i.e. $A \stackrel{=}{=} T \oplus D$ where $T$ is the torsion subgroup of $A$. Then there is $\delta \in E$ and $\nu>\delta$ so that $A_{\delta}=\left(T \cap A_{\delta}\right)+\left(D \cap A_{\delta}\right)$ and $A_{\nu}=$ $\left(T \cap A_{\nu}\right)+\left(D \cap A_{\nu}\right)$. So

$$
A_{\nu} / A_{\delta} \cong\left(T \cap A_{\nu}\right) /\left(T \cap A_{\delta}\right) \oplus\left(D \cap A_{\nu}\right) /\left(D \cap A_{\delta}\right) .
$$

So $A_{\nu} / A_{\delta}$ is split but $A_{\nu} / A_{\delta} \cong G+C$ where $C$ is $\Sigma$-cyclic. So we have a contradiction.

\section{REFERENCES}

[P] K. Devlin, A Yorkshire man's guide to proper forcing, Surveys in Set Theory, London Math. Soc. Lecture Notes No. 87, Cambridge Univ. Press, 1983, pp. 60-115.

[E1] P. Eklof, Infinitary equivalence of abelian groups, Fund. Math. 81 (1974), 305-314.

[E2] $\_$, Set theoretic methods in homological algebra and Abelian groups, Presses Univ. Montréal, 1980.

[E3] _ The structure of $\omega_{1}$-separable groups, Trans. Amer. Math. Soc. 279 (1983), 497-523.

[EM1] P. Eklof and A. Mekler, On constructing indecomposable groups in L, J. Algebra 49 (1977), 96-103.

[EM2] _ On endomorphism rings of $\omega_{1}$-separable primary groups, Lecture Notes in Math., vol. 1006, Springer-Verlag, Berlin and New York, 1983, pp. 320-339.

[F] L. Fuchs, Abelian groups, Hungarian Academy of Sciences, Budapest, 1958.

[F2] _ On $p^{\omega+1}$-projective Abelian p-groups, Publ. Math. Debrecen. 23 (1976), 309-313.

[FI] L. Fuchs and J. Irwin, On $p^{\omega+1}$-projective p-groups, Proc. London Math. Soc. 30 (1975), 459-470.

[H] P. Hill, On the decomposition of groups, Canad. J. Math. 21 (1969), 762-768. 
[Meg 1] C. Megibben, Crawley's problem on the unique $\omega_{1}$-elongation of p-group is undecidable, Pacific J. Math. 107 (1983), 205-212.

[Meg 2] _ , $\omega_{1}$-separable p-groups (preprint).

[M1] A. Mekler, How to construct almost free groups, Canad. J. Math. 32 (1980), 1206-1228.

[M2] _ Shelah's Whitehead groups and CH, Rocky Mountain J. Math. 12 (1982), 272-278.

[M3] _ _ Proper forcing and Abelian groups, Abelian Group Theory, Lecture Notes in Math., vol. 1006, Springer-Verlag, Berlin and New York, 1983, pp. 285-303.

[M4] _ c.c.c. forcing without combinatorics, J. Symbolic Logic 49 (1984), 830-832.

[S1] S. Shelah, Infinite Abelian groups, Whitehead problem and some constructions, Israel J. Math. 18 (1974), 243-256.

[S2] _ Proper forcing, Lecture Notes in Math., vol. 940, Springer-Verlag, Berlin and New York, 1982.

Department of MAThematics and Statistics, Simon Fraser UNiVERSity, BuRNABY, BRITISH COLUMBIA, CANADA V5A 1S6 